\title{
Calibration of the TA Fluorescence Detectors with Electron Light Source
}

\author{
Bokkyun Shin*1, Tatsunobu Shibata ${ }^{2}$, Dmitri Ivanov ${ }^{3}$, Masaki Fukushima ${ }^{4}$, Gordon \\ Thomson $^{3}$, John Matthews ${ }^{3}$, B.G. Cheon ${ }^{1}$, Hiroyuki Sagawa ${ }^{4}$, for the Telescope \\ Array Collaboration ${ }^{\dagger}$ \\ ${ }^{1}$ Department of Physics, Hanyang University, 222 Wangsimni-ro Seongdung-gu \\ Seoul,133-791, Korea \\ ${ }^{2}$ High Energy Accelerator Research organization (KEK), Japan \\ ${ }^{3}$ High Energy Astrophysics Institute and Department of Physics and Astronomy, University of \\ Utah, Salt Lake City, UT, USA \\ ${ }^{4}$ Institute for Cosmic Ray Research, University of Tokyo, Kashiwa, Chiba, Japan \\ E-mail: bkshin@hanyang.ac.kr
}

The Electron Light Source (ELS) is a linear accelerator used to perform energy calibration of the fluorescence detectors (FD) in the Telescope Array experiment. The ELS shoots a beam of 40 $\mathrm{MeV}$ electrons into the atmosphere $100 \mathrm{~m}$ in front of the Black Rock Mesa FD. Air fluorescence light is detected from nitrogen molecule excitation by the ELS electron beam. An end-to-end calibration from generation of fluorescence by air to detection of fluorescence photon by FD PMT camera is achieved. We present the calibration method and the comparison between beam data and Monte Carlo simulation.

The 34th International Cosmic Ray Conference,

30 July- 6 August, 2015

The Hague, The Netherlands

\footnotetext{
* Speaker.

${ }^{\dagger}$ Full author list and Acknowledgements: http://www.telescopearray.org/images/papers/ICRC2015-authorlist.pdf
} 


\section{Introduction}

The Telescope Array is an observatory for ultra high energy cosmic rays (UHECR) located in Utah, USA [1]. The Telescope Array experiment has three stations of Fluorescence Detectors (FD) [2] and 507 surface detectors arranged on a grid of $1.2 \mathrm{~km}$ spacing [3]. An FD station consists of 12 or 14 telescopes each of which has a $3.3 \mathrm{~m}$ radius spherical mirror to collect fluorescence light and focus it on a camera consisting of a $16 \times 16$ array of photomultiplier tubes (PMT). The fluorescence photons are emitted by nitrogen molecules excited by charged particles in cosmic ray air showers.

The ELS is an electron linear accelerator built for an end to end calibration for the TA FDs. It was developed at the KEK laboratory in 2008 [4] and deployed $100 \mathrm{~m}$ in front the FD station at Black Rock Mesa (BRM) [5]. Figure 1 shows the position of the ELS in relation to the BRM $\mathrm{FD}$, and a picture of the ELS beam taken with the BRM FD cameras. The ELS fires a vertical 40 $\mathrm{MeV}$ electron beam of duration $1 \mu \mathrm{s}$ at a repetition rate of $0.5 \mathrm{~Hz}$. Briefly, the ELS consists of RF cavities that accelerate the electron beam, quadrupole magnets for focusing, a bending magnet to direct the beam upward into the sky, and collimators, Faraday cups, and pickup coils called core monitors.

The end to end calibration for the energy scale of the BRM FD is achieved by comparing the number of photoelectrons detected by the FD to the number predicted using the following inputs: the beam parameters of the ELS, measurements of the yield of fluorescence photons per MeV from energy deposited in the air by ELS electrons, and the event reconstruction programs of the TA collaboration. A Monte Carlo simulation of the ELS was performed, where the geometry of the accelerator and the beam parameters were used to predict the spatial distribution of energy loss in the atmosphere, including backgrounds from gamma rays and low energy electrons. This distribution was used as the input to TA FD reconstruction programs to predict the number of photoelectrons seen by the BRM FD telescopes. Three different experimental measurements of the fluorescence yield (see Section 5) were used in this prediction.

The first ELS operation occurred in 2010; regular data collection for FD calibration began in 2011, and the first results were reported at the 33rd International Cosmic Ray Conference [6].

The ELS has also been a resource for research on radio techniques in cosmic ray detection, such as molecular bremsstrahlung, radio wave scattering from air showers [7], and the study of the Askaryan effect in ice [8][9][10].

\section{Measurement of Beam Parameters}

We measured three beam parameters: beam charge, beam energy distribution and beam emittance. The beam charge was measured by the same method described in reference [6]. This, and the additional two parameters were measured in March, October and November 2014. We will describe each measurement here.

The beam charge was measured with a Core Monitor (CM) which is installed near the point of beam exit of the accelerator. The CM measures the beam charge by induced current in a coil of 25 turns, which is read out by integration of the waveform by an oscilloscope. The CM was calibrated by a direct measurement using two Faraday Cups (FC) [6] inserted onto the beam axis $12 \mathrm{~cm}$ above the beam exit window during special calibration runs. 


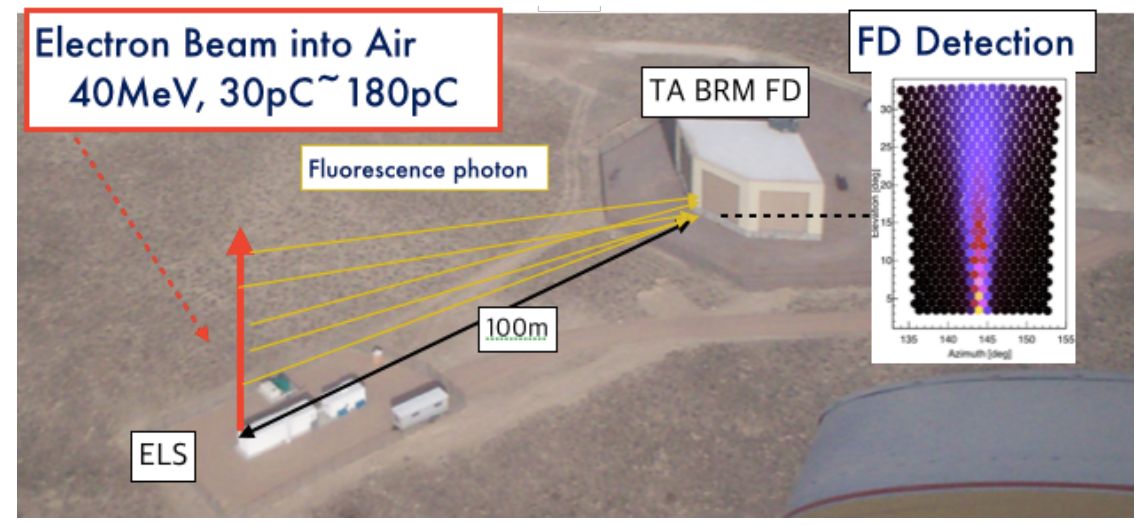

Figure 1: ELS position in relation to the TA BRM FD, and (inset) a picture of the of ELS beam taken with two telescopes of the BRM FD.

The two FC's, which consist of $60 \mathrm{~mm}$ depth of copper, had different read-out devices. One FC is read out with a Coulomb meter (Q-meter) which accumulates charge over a 2 second period, and the other is read out with an oscilloscope. Figure 2 shows a CM vs. FC calibration scatter plot. Black points use the Q-meter readout, and red points use the oscilloscope readout. The two measurements agree within 3.3\%, and we use the average of the two slopes for CM calibration.
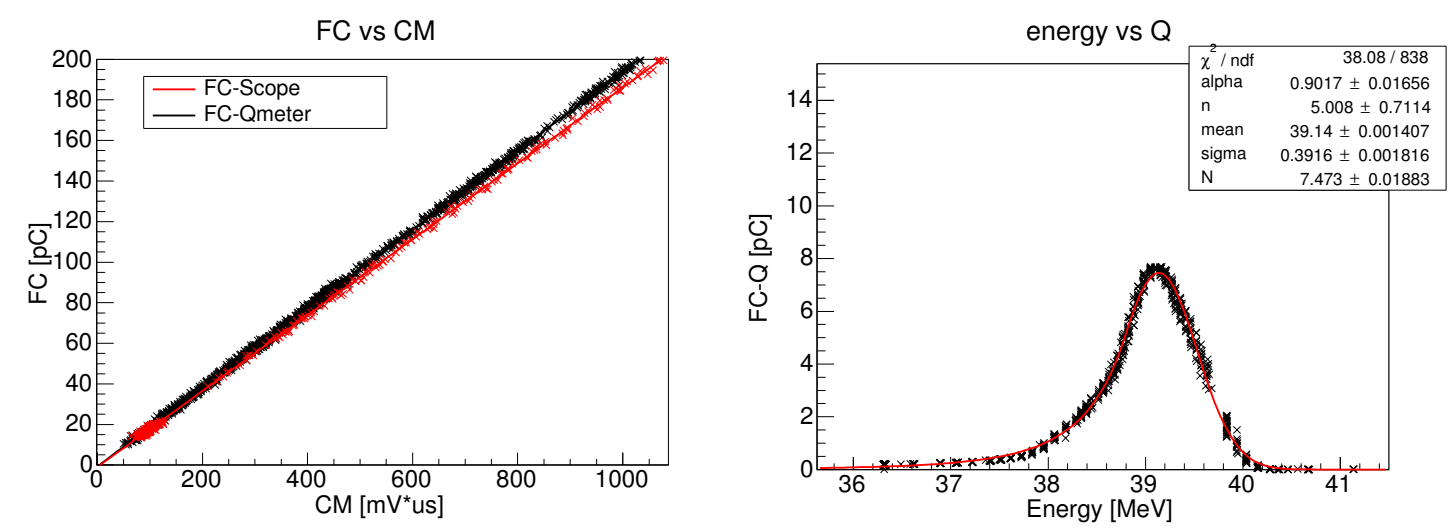

Figure 2: Left: Scatter plot of CM vs. FC readouts in a calibration run on March 22nd 2014. The FC is inserted into the beam during this run. Red dots represent the measurement with oscilloscope readout of the FC, and Black is for Q-meter readout. Right: ELS beam energy distribution before the bending magnet. The fitting result using the Crystal-ball function [11] is shown as the red line.

An electron energy distribution can be measured with the $90^{\circ}$ Bending Magnet (BM) which has a radius of curvature of $220 \mathrm{~mm}$, with the beam focused on a collimator of gap typically $3 \mathrm{~mm}$. Changing the magnetic field of the BM and measuring the beam charge with the FC resulted in Figure 2 (Right), which shows the measured energy distribution. It has a long tail at lower energies. The source of the tail is de-accelerated electrons which will be dumped in the BM and the collimator. Our Monte Carlo simulation of the ELS uses GEANT [12] to predict the fluxes of background electrons and gamma-rays which are shot into the sky.

The beam spread is also important for precise estimation of the contribution of the backgrounds. The beam spread was analyzed by measurement of the emittance and Twiss parameters 
of the beam. The emittance $(\varepsilon)$ and Twiss parameters $(\alpha, \beta, \gamma)$ are related with particle position $(X)$ and direction $\left(X^{\prime}\right)$ by $\varepsilon=\beta X^{2}-\alpha X X^{\prime}+\gamma X^{\prime 2}$. The emittance and Twiss parameters were measured by scanning the quadrupole magnets $(\mathrm{QM})$ current and determining the beam size using a beam spot screen $15 \mathrm{~cm}$ downsteam of the second QM. The result of the beam spread measurement is shown in Table 1.

\begin{tabular}{|c|c|c|c|c|}
\hline Axis & $\alpha$ & $\beta[\mathrm{rad} / \mathrm{m}]$ & $\gamma[\mathrm{m} / \mathrm{rad}]$ & $\varepsilon[\mathrm{m} \mathrm{rad}]$ \\
\hline Vertical & -0.265 & 1.320 & 0.811 & $8.076 \mathrm{e}-06$ \\
Horizontal & -0.369 & 3.525 & 0.322 & $2.743 \mathrm{e}-06$ \\
\hline
\end{tabular}

Table 1: Emittance and Twiss parameters that characterize the ELS beam.

\section{ELS Beam Detection by the TA BRM FD}

The AF photons from the ELS beam are detected by two of the BRM FD telescopes. The total field of view (FoV) of the two telescopes is $3^{\circ}-33^{\circ}$ in elevation, and $18^{\circ}$ in azimuth. A typical ELS data-set was taken with about 700 ELS shots with beam charge varied from $20 \mathrm{pC}$ to $160 \mathrm{pC}$, in about 20 minutes of operation. The Figure 3 (left) is an ELS event map of the two telescopes, and the color axis shows the integrated number of FADC counts.
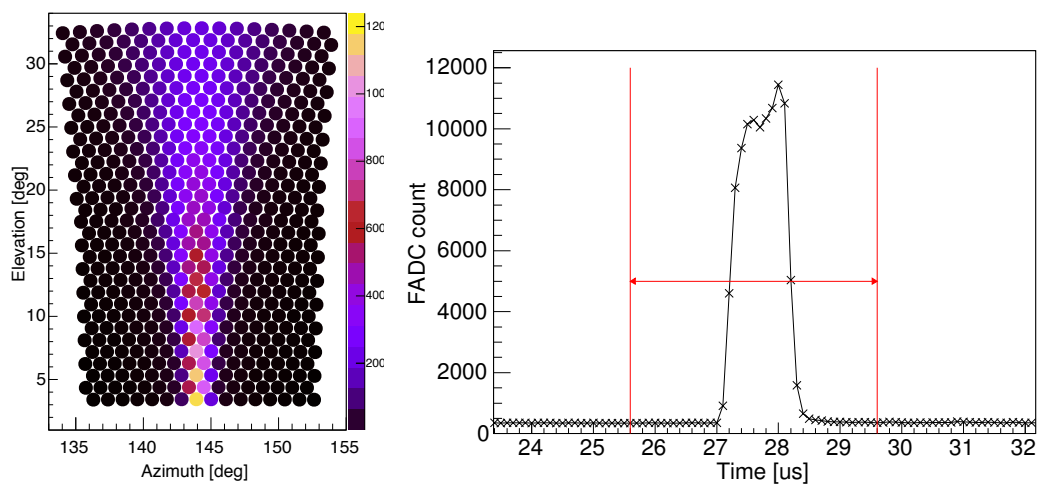

Figure 3: Left: Event map of ELS by 512 PMTs which is made accumulated over 700 ELS shots in one run. Right: FADC waveform of the bottom center PMT. Integration of the FADC signal is performed over the $4 \mu$ s around FADC peak (in the range of the red arrow).

\section{Analysis - definition of parameters}

We define a variable $S$ as an integrated number of FADC counts normalized to the unit amount of ELS beam charge,

$$
S=\Sigma F A D C / Q
$$

where $\Sigma F A D C$ [FADC counts] is obtained from the FD waveform as shown in Figure 3 (Right) after subtracting the pedestal, integrating over the signal time range, and summing over all the 512 
PMTs in the two BRM telescopes that see the ELS beam. $Q$ is defined as the beam charge [pC] measured by the core monitor.

The beam measurement by the FC is destructive, so we measure the $Q$ of an ELS run by using the non-destructive core monitor (CM) measuring the beam current of each ELS shot. The CM is used as a relative monitor with its absolute sensitivity calibrated by dedicated calibration runs using the FC before each ELS run. One of the results of the FC-CM calibration run is shown in Figure 2.

The $Q$ for the ELS simulation is obtained by making a separate MC run with the FC installed in the beam line (and destroying the beam at the FC).

Each ELS run is composed of approximately $700 \mathrm{ELS}$ shots into the air at $0.5 \mathrm{~Hz}$. The intensity of each shot is slowly changed during a run usually covering the range from $20 \mathrm{pC}$ to $200 \mathrm{pC}$. The scatter plot of $\Sigma F A D C$ and $Q$ obtained for each shot is shown in Figure 4. The value of $S$ is obtained as the slope of a linear fit to the scatter plot.

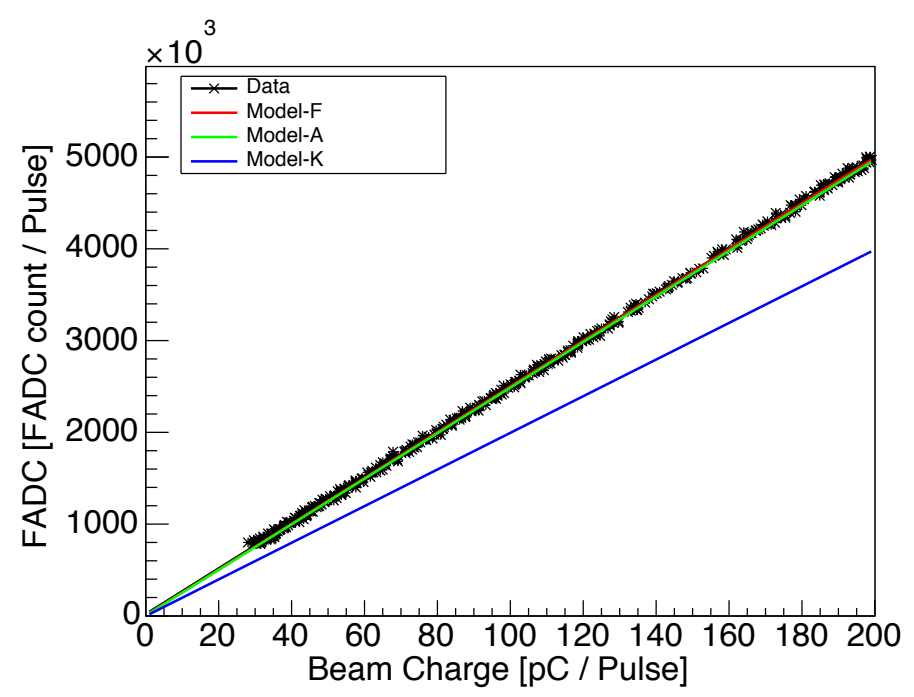

Figure 4: Scatter plot of beam charge vs. FADC for one ELS data-set. The data and a fit to the data are shown in black, and simulation results with three AFY models are shown as red, green, and blue lines.

An ELS simulation run is composed of two independent MC runs: one without the FC in the beam line to estimate the $\Sigma F A D C$ generated from the energy deposit in the atmosphere, and another with the FC in the beam line to estimate the $Q$ of the same ELS beam. Each MC run is composed of 100k electrons generated (according to the energy distribution and emittance as mentioned in Section 2) at a point just before the first quadrupole magnet. Approximately 10k electrons peaked near $40 \mathrm{MeV}$, and 10k photons significantly below $1 \mathrm{MeV}$, reach the end of the vertical beam line and are injected into the air, or into the FC.

The value of $\Sigma F A D C$ and $Q$ are summed over all the simulated events (100k electrons) and $S$ is calculated as the ratio of $\Sigma F A D C$ over $Q$. Neither the time structure nor the FADC waveform of each MC event was simulated. We used three different air fluorescence yield (AFY) models in the FD simulation. The details of the AFY models are described in section 5. We define a ratio $R$ of the measured $S$ and the value of $S$ expected from the simulation for comparison of data to the AFY models as,

$$
R_{A F Y}=S_{D A T A} / S_{M C}^{A F Y}
$$




\section{AFY models}

We used three AFY models to compare with the ELS data taken in 2014: model-F is based on the FLASH experiment [13]; model-K is based on the Kakimoto et al. measurement [14]; and model-A is based on the AIRFLY experiment [15].

Model-F uses the AF spectrum covering 300-420nm measured by FLASH [13]. For model-F, we take the FLASH AFY analyzed using a density and temperature dependence from the formula found in Kakimoto et al. [14].

For model-K, we took the equation and parameters of Kakimoto et al. [14]. The total AFY is then increased by $2.1 \%$ to the value corresponding to the range of $300-420 \mathrm{~nm}$, and distributed for each line using the FLASH spectrum. The dependence on the relative humidity at the time of an ELS run is not considered in either model-F or model-K.

For the AFY in model-A, we used an AFY of 5.61 photons per MeV measured for the $337 \mathrm{~nm}$ line in dry air of $1013 \mathrm{hPa}$ and 293K [15]. The AFY of each line in the 300-420 nm range is obtained by using the AF spectrum of AIRFLY [16]. For ELS simulation, the dependence on pressure, temperature, and humidity is taken into account for each spectral line using the reference model formula which was reported at UHECR2012 [17].

\section{Result and Discussion}

We analyzed 6 ELS data-sets which also included measurements of the beam's energy distribution and spatial spread. In the Monte Carlo simulation, we calculated FADC values expected using the three AFY models and detector parameters relevant at the data-collection times. Figure 4 is a scatter plot of FADC sums vs. beam charge $Q$ for the first run of 2014. The data are denoted by black dots along with a linear fitting line, and lines of red, green, and blue are result of simulations with the three AFY models. Table 2 shows the result of the 6 ELS data-sets with atmospheric conditions and comparisons between data and the three AFY models.

The average results for R are 1.008 for Model-F, 1.257 for Model-K and 1.019 for ModelA. The statistic errors of $\mathrm{R}$ are estimated to be $0.1 \%$ from simulation and $0.01 \%$ from data. The systematic uncertainty of $\mathrm{R}$ is estimated to be $7.9 \%$. The breakdown of the systematic uncertainties are given in Table 3.

\begin{tabular}{|c|c|c|c|c|c|c|}
\hline \multirow{2}{*}{ Run } & \multicolumn{3}{|c|}{ Atmospheric condition } & \multicolumn{3}{c|}{$R_{A F Y}$} \\
\cline { 2 - 7 } & $\mathrm{T}\left[{ }^{\circ} \mathrm{C}\right]$ & $\mathrm{p}[\mathrm{hPa}]$ & $\mathrm{H}[\%]$ & Model-F & Model-K & Model-A \\
\hline 1 & 7.3 & 855.1 & 29.2 & 0.998 & 1.245 & 1.002 \\
2 & 6.8 & 861.8 & 16.8 & 0.996 & 1.245 & 0.991 \\
3 & 0.0 & 865.1 & 47.9 & 1.004 & 1.264 & 1.016 \\
4 & 0.4 & 864.2 & 47.5 & 1.010 & 1.248 & 1.025 \\
5 & -1.5 & 864.6 & 63.1 & 1.025 & 1.275 & 1.041 \\
6 & -5.0 & 864.3 & 71.3 & 1.017 & 1.267 & 1.037 \\
\hline \multicolumn{3}{|c|}{ Average } & 1.008 & 1.257 & 1.019 \\
\hline
\end{tabular}

Table 2: Result of R with three different AFY models. 
We made 6 ELS runs in 2014 to make an end-to-end (from energy deposit in the air to FADC counts) calibration of two TA telescopes at the BRM site. The ELS data was analyzed by the same programs used for the TA air shower analysis. The same geometrical, optical, and calibration parameters have been maintained in the simulation and the data analysis programs. The ELS data is compared with the expectation using three different AFY models: model-F, -K and -A from the FLASH, Kakimoto et al., and AIRFLY experiments. A comparison between the data and the simulation is well represented by the ratio parameter R between the ELS data and the MC expectation.

We found that the features of the ELS data are well reproduced by the ELS simulation using the air fluorescence model-F and model-A, with $\mathrm{R}=1.01$ and 1.02 respectively. The systematic uncertainty in the comparison of TA BRM FD data to the ELS simulation is estimated to be $7.9 \%$ as shown in Table 3.

Model-K, on the other hand, gives $\mathrm{R}=1.26$. Considering the $10 \%$ systematic uncertainty in the AFY measurement of Kakimoto et al., and the $10 \%$ uncertainty estimated in the TA overall telescope sensitivity [18], we conclude that using model-K we cannot explain the ELS data.

\begin{tabular}{|ll|l|l|}
\hline 1. & Q in ELS data & $3.3 \%$ & Difference in two FC measurements \\
2. & Soft photon background & $1.5 \%$ & Dependence in ELS beam parameters \\
3. & Cherenkov photon background & $0.4 \%$ & Estimated and not subtracted \\
4. & FADC of simulation & $4.0 \%$ & Difference by simulation package \\
5. & P, T and h at BRM & $0.5 \%$ & Difference of R by ambiguity of P,T and h \\
6. & IFADC in ELS MC & $5.5 \%$ & Difference by pixel summation region \\
7. & Telescope parameters & $1.6 \%$ & Time, temperature dependence and others \\
\hline & TOTAL & $7.9 \%$ & quadratic sum of 1-7 \\
\hline
\end{tabular}

Table 3: Systematic uncertainties of $\mathrm{R}$

\section{References}

[1] H. Sagawa et al., Proc. 33rd International Cosmic Ray Conference, Rio de Janeiro 2013

[2] H. Tokuno et al., Nuclear Instruments and Methods in Physics Research A 676 (2012) 54-65

[3] T. Abu-Zayyad et al., Nuclear Instruments and Methods in Physics Research A 689 (2012) 87-97

[4] T. Shibata et al., Nuclear Instruments and Methods in Physics Research A 597 (2008) 61-66

[5] T. Shibata et al., Proc. 32nd International Cosmic Ray Conference, Beijing, China 2011

[6] T. Shibata et al., Proc. 33rd International Cosmic Ray Conference, Rio de Janeiro 2013

[7] D. Ikeda et al., Proc. 34th International Cosmic Ray Conference, The Hague, The Netherlands 2015, in these proceedings

[8] P. Allison et al., Astroparticle Physics 35 (2012) 457-477

[9] S. Ueyama et al., 34th International Cosmic Ray Conference, The Hague, The Netherlands 2015, in these proceedings 
[10] T. Yamamoto et al., 34th International Cosmic Ray Conference, The Hague, The Netherlands 2015, in these proceedings

[11] T.Skwarnicki, Ph.D Thesis, DESY F31-86-02(1986)

[12] S. Agostinelli et al., Nuclear Instruments and Methods in Physics Research A 506 (2003) 250-303

[13] R. Abbasi et al., Astroparticle Physics 29 (2008) 77-86

[14] F .Kakimoto et al., Nuclear Instruments and Methods in Physics Research A 372 (1996) 527-533

[15] M. Ave et al., Astroparticle Physics 42 (2013) 90-102

[16] M. Ave et al., Nuclear Instruments and Methods in Physics Research A 597 (2008) 41

[17] B. Keilhauer et al., EPJ Web of Conferences 53, 01010 (2013)

[18] T. Abu-Zayyad et al., Astroparticle Physics 61 (2015) 93-101 